\title{
Pain and Care: Interventions in Daily Nursing Practice
}

\author{
Lambrini Kourkouta ${ }^{1}$, Areti Tsaloglidou ${ }^{1}$, Konstantinos Tsaras $^{2}$, Konstantinos Koukourikos ${ }^{1}$, Christos F. \\ Kleisiaris $^{3}$, Evangelos C. Fradelos ${ }^{4}$ and Ioanna V. Papathanasiou ${ }^{2}$ \\ 1. Nursing Department, Alexander Technological Educational Institute of Thessaloniki, Thessaloniki 57400, Greece \\ 2. Nursing Department, Technological Educational Institute of Thessaly, Larissa 41110, Greece \\ 3. Nursing Department, Technological Educational Institute of Crete, Heraklion 71500, Greece \\ 4. State Mental Hospital of Attica "Daphne”, Athens 12462, Greece
}

\begin{abstract}
Pain is a common symptom in humans, which differs significantly depending on their status of health. This review lists the specific features of pain that are necessary for its evaluation and treatment by the health professionals. Extensive review of the recent literature was conducted in electronic databases (PubMed, Medline and Iatrotek) and scientific journals in Greek and English. Pain is perceived as any uncomfortable feeling, and emotional experience that occurs anywhere in the body when there is some kind of injury. The assessment of pain in humans is a complex and demanding process. Pain should be treated early with appropriate pharmaceutical or other treatment. Each pain has a different cause and treatment. The objective of health professionals' interventions is to cure or at least reduce pain and to relieve those who are in pain.
\end{abstract}

Key words: Pain, nursing care, nursing interventions, nursing practice.

\section{Introduction}

Every human being, from the moment that comes into this world, meets with the pain, which is closely related to his/her life. Pain affects humans regardless of their gender, age, economic status and cultural behavior. The human, therefore, is born with pain. $\mathrm{He} /$ she gains this temporary life with pain and leaves from this world with pain [1, 2].

The occurrence of pain in human life is a sign of intense questioning for the people of all the eras. The pain has never ceased and will never stop to exist. It will always coexist with human life. This is the involuntary companion of every human being. In all the eras of humanity, from the first social groups until today's society, an attempt is being made in order the best possible and prompt treatment of pain to be provided [3].

Taking into consideration the perception that each pain has a different cause and treatment and that every

Corresponding author: Lambrini Kourkouta, Ph.D., professor, research fields: history of nursing, ethics and deontology. patient who is in pain is a separate psychosomatic entity, an attempt is being made in order the patient who has the problem to understand it, to be adapted to the possibilities of a treatment and not to be withdrawn from everyday life's activities [4].

Pain is an unpleasant feeling with strong personal characteristics. It is possible to absorb the thought of an individual, to influence his activities and to change all his life [3].

The IASP (International Association for the Study of Pain) [5] gives the following definition for the pain, which has been widely prevailed, "an unpleasant sensory and emotional experience associated with actual or potential tissue damage and is expressed in terms that suggest the character and the extent of the damage" [5].

Each pain affects the lives of not only the patient but also the family and the environment in which he lives and is socialized. Pain relief is a priority in the care of the patient [6]. According to a literature review of electronic databases from 2008 to 2010, a percentage of $78 \%$ of people entering the outpatient 
clinics mentions that are in pain, while $29 \%$ of the general population's absences from work is due to pain [7].

Moreover, despite the availability of effective treatments for the pain, there are many obstacles to its effective management, resulting in a high percentage of patients who are not able to gain its control [8].

The purpose of this study is to present the forms and types of pain and their treatment by the health care professionals, promoting in this way, the quality of patients' life.

\section{Material and Methods}

A review of the recent literature was conducted in authoritative databases, such as PubMed, Medline and Iatrotek. Criterion selection of the studies was the Greek and English, using the following key words: pain, nurse and disease, and treatment.

\section{Talking about Pain}

Pain is the second symptom in frequency after fever. This has important implications in both the professional and social life of people [9]. The pain is an unpleasant, more than the tolerance level, feeling which usually results from a very severe or catastrophic bodily stimulus. It is experienced subjectively as it is influenced by the state of the brain in which the sensation and the pain as its extension are formed $[10,11]$.

The pain receptors called nociceptors. They are free nerve endings and are found in all body tissues outside the brain. The types of receptors are neuro-sensitive, heat-sensitive and chemo-sensitive. The pain receptors, instead of being familiar, become sensitive $[12,13]$.

\subsection{Types of Pain}

For understanding the pain inducing mechanism, it is necessary for the pain to be distinguished in two qualitatively separate entities:

(1) Physiological pain: It is responding to the operation of normal sensations. It is a normal protective mechanism that removes us from even the temporarily harmful factors [14]. In everyday life, every man, who is touching cold or hot objects or is accepting pressure from intense stimulus, sting or rake, has multiple opportunities to understand what "physiological pain" means and to assess his tolerance capabilities against it $[10,13]$;

(2) Pathological pain: It is the sensation caused by either an inflammatory response which accompanies a tissue trauma, or nervous system damage. Therefore, it is divided into inflammatory and neurological pain.

Pain is also divided into acute or chronic pain $[12,14]$ :

(1) Acute pain: It is pain of short duration and usually its cause can be identified. In fact, acute pain acts like danger signal for a threatened injury or for an injury that has already occurred. It is the physiological reaction to a harmful or dangerous stimulus [15].

The causes give rise to tissue damage, which is reversible. From a biological perspective it may have warning, diagnostic or therapeutic value $[16,17]$;

(2) Chronic pain: It is often independent from the original cause. The cause of pain may no longer exist but the over-excited nerves may still report pain to the brain $[18,19]$.

Chronic pain is one that lasts for more than three months, or the pain that insists for more than one month of the usual course of an acute disease or more than a reasonable time required for wound healing $[18,20]$.

Furthermore, pain is also divided into rapid and slow pain:

(1) The rapid pain occurs in $0.1 \mathrm{~s}$ after the application of a painful stimulus. Pain of this type is felt by inserting the needle into the skin, or during the skin incision with a knife, as well as when an acid burn of the skin occurs $[12,17]$;

(2) The slow pain, from the other side, begins to be felt after $1 \mathrm{~s}$ or more, and then its intensity increases slowly for several seconds, and in many cases even for several minutes. Pain of this type is usually associated 
with tissue destruction $[18,20]$.

Other types of pain are also referred to the literature. Each of them has its own characteristics, which are described below:

(1) The somatic pain comes from muscles, bones, skin, subcutaneous tissue and peritoneal and it is led by $\mathrm{A}_{\delta}$ and $\mathrm{C}$ fibers. It is distributed in the corresponding dermatome of the stimulated root. Pain is sharp, clear and localized [12]. It presents a clear localization and normally is demonstrated by the patient's finger. It can be classified as acute, intense and stabbing "like a stab". It is getting worse by movement, coughing, deep breathing movements and the gait and for this reason the patient tries to remain in the same position [10];

(2) Visceral pain comes from the viscera and is conducted with $\mathrm{C}$ fibers and few $\mathrm{A}_{\delta}$ fibers. It is distributed to the respective elements of the ANS (Autonomic Nervous System). It is blunt pain, not being localized. Visceral pain presents vague localization and is demonstrated by the patient's palm. It is usually aching, blunt and diffuse, and it extends from the point of failure to a larger area of the body $[12,19]$;

(3) Neuropathic pain follows the damage to the peripheral or central nervous system. There are no specific receptors involved and the pain is caused by the impairment of nerve cells. Where there is a nerve pain, the pain is generated within the nervous system itself. In the case of so-called "sympathetic" pain, the pain is due to over-activity of the peripheral or central nervous mechanisms [12, 13];

(4) Reflexive pain is usually the phenomenon of the visceral pain, which is located in another area of the body, sufficiently distant from the affected organ. A classic example is the pain of myocardial infarction which reflects behind the sternum, lower jaw, left shoulder and arm [3, 13];

(5) Psychogenic pain is not experienced differently by the so-called organic pain. For example, when tension headaches occur, the pain is the result of muscle contraction (organic base), which happens as a consequence of stress and negative emotions (psychological basis) [1, 6].

The phantom limb pain occurs after limb amputation or even after cerebral palsy or quadriplegia. It is persistent and can last from months to years after the healing of traumatic surface of the abutment. It is attributed to psychological causes $[3,19]$.

\subsection{Evaluation and Measurement of Pain}

Pain's assessment is necessary for the evaluation of the magnitude and effectiveness of treatment. Most of the Pain Response Centers use a special form questionnaire and examination sheet, in order to have a complete picture of the patient's situation. According to it, history, physical examination and laboratory tests are received and assessed, where possible [21].

The pain measurement scales also give indications of the intensity and the duration of pain, such as the following ones $[7,15]$ :

- Verbal description scale (verbal descriptor scale): Five words are commonly used: medium, annoying, obsessive, terrible and unbearable pain. Patients usually choose the middle words;

- Numbers assessment scale/visual analogue scale (numeric rating scale/visual analog scale): A numerical scale from 0 (no pain) to 10 (the worst pain) is used. It is simple and easy to use;

- Table with face shapes with a smile to crying (continuum of smiling to crying faces). It can be used by children.

Pain is usually being investigated experimentally by recording the hot stimulus tolerance (the guinea pig is forced to walk on a hot surface) [22].

\subsection{Treatment of Pain}

Each pain has a different cause and treatment. The patient who is in pain is a separate psychosomatic entity. Thus, every health care professional must try to 
help him realize his problem, to adapt to the possibilities of the treatment and not to withdraw himself from the everyday life's activities $[6,10]$.

A basic prerequisite for the selection of a suitable analgesic is the knowledge of its pharmacokinetic and pharmacodynamic properties, in order to achieve a constant concentration - density of it to the plasma and stable levels of analgesia. The types of drugs used to treat pain are listed as follows [16, 22]:

- analgesics with peripheral action;

- analgesics centrally-acting;

- non-specific analgesic drugs;

- sedatives and anti-anxiety drugs.

Analgesics with peripheral action are more effective in mild to moderate inflammatory pain, postoperative pain, traumatic pain, pain of arthritis and cancer pain. The analgesic effect is enhanced when used in combination with opioids or other adjuvant therapy [23]. Their adverse effects are mainly related to digestive and blood system. The most commonly used are the following [24]:

- carboxylic acids (salicylates, acetate, propionate and anthranilic acids);

- pyrazoles;

- oxicams.

The centrally acting analgesics are also called opioid analgesics, because as they occupy the endogenous opioid receptors (enkephalin, endorphin and dinorphin) in the brain and spinal cord, they mimic their action. The selection of a suitable opioid corresponding dose and route of administration, depends on the kind of pain [16, 24].

The opioid therapy is indicated if the chronic cancer or non-malignant pain is not responding to the treatment with non-opiates. The utility of these drugs is limited as much as possible at low doses, because of their toxicity, side effects, physical dependence and addiction or withdrawal risk $[17,25]$.

Physical dependence and withdrawal symptoms can be prevented by careful monitoring, gradual discontinuation of the substance and gradual reduction of drug doses $[9,18]$. The psychological dependence is rare when there is proper administration of the drug. The pseudo-addiction is a form of behavior by which the patient is looking for the drug in an attempt to relieve the pain and it can be regarded as a normal addiction. It is often the result of an uncontrolled pain due to inadequate treatment $[24,26]$.

Non-specific analgesics according to their mode of action are distinguished in the following categories [24]:

- Tricyclic antidepressants: They relieve the depression and stress that accompany chronic pain;

- Antispasmodics: They are administered to trigeminal neuralgia, peripheral neuropathies, post-traumatic injury syndromes of the spinal cord, and to phantom limb;

- Antiarrhythmics: They are mainly used for the treatment of neuropathic pain;

- Anti-serotonin and antihistamines: They act against serotonin and histamine that are released distally from the platelets and mastocytes;

- Corticosteroids: They inhibit the formation. Prostaglandins are used only in chronic inflammatory conditions;

- Muscle relaxants: They cause moderate depression of the CNS (Central Nervous System) and inhibition of peripheral reflexes trends;

- Local anesthetics: In systemic administration, they are mainly used for the treatment of neuropathic pain. Topical administration restricts secondary hyperalgesia;

Calcitonin. It is used to treat bone pain from primary or metastatic tumor foci. Its action is due to an increase of beta-endorphin.

Sedatives and anti-anxiety drugs are administered to calm the patient and relieve his anxiety and fear which is created by the untreated pain, as well as to provide sleep. Their adverse effects may lead to a paradoxical increase of anxiety and insomnia, mental concentration, acceptance inability, and dependence. It is required appropriate dose selection and 
separation $[20,24]$.

Occasionally, the maximum analgesic effect is obtained 4 6 weeks after the attainment of the therapeutic level. Patients should be educated about the possible delay in the action of these drugs until the right combination, the one that will benefit him, is found $[26,27]$.

\subsection{Nursing Treatment of Pain}

When the pain is evaluated, except from the clinical examination, useful data are also the communication with the patient and his environment, the study of his/her health history and the diagnostic findings [7]. The diagnosis of pain's nature helps in choosing the most appropriate nursing interventions for the alleviation and minimization of the adverse effects.

Many health care professionals and academics tried to explain the paradox of pain. Medical and Nursing science examining the pathophysiology of pain tries to cope with the pain. An attempt is being made to cure or at least reduce pain and relieve the persons who are in pain by various means [10]. The availability of drugs in combination with the health professionals' education, and the implementation of an appropriate protocol that takes into account the pharmacokinetics of drugs and the subjective satisfaction of the patients, as well as the interpretation of their statements, will lead to the separation of pain from a medical condition and, therefore, to a more effective treatment regimen [7].

The main points of nursing diagnosis of pain are the following [10]:

- determination of the patient's reaction to pain;

- evaluation of pain points, such as anxiety, sweating and paleness;

- evaluation of the factors that appear to exacerbate or reduce pain.

The criteria of the evaluation of nursing intervention results focus on the following [10, 28]:

- The patient reports that the pain has been reduced or eliminated after the application of analgesic instruments or measures;

- Daily activities are growing progressively, he/she also states that has a better sleep;

- He/she is able to use non-pharmacological analgesia techniques.

Patients commonly do not understand the reason of pain. Therefore, nurses should provide the right time so that individuals are adapted to the new situation. Additionally, administration of drugs before any painful operation and before the intensification of pain is effective in treating pain, as the intense and prolonged pain more slowly recede [10].

The social environment should also support the patient. In many cases, the patient who is in pain feels socially isolated, because none of his/her relatives or the health personnel is able to truly communicate with him/her or they pretend regarding his/her health situation. The stress caused by the patients' condition, makes their relatives and health personnel be removed, in order to protect their own psychology $[17,27]$.

Additionally, nurses support psychologically both patients and their families, offering not only information about the pain, but also proposing ways to address the problems that they encounter [17, 29].

In the battle against pain used in during different stages of human history, a variety of therapeutic methods are used [30]. A key component is also the communication between the nurse and the patient. Communication is a vital element in nursing practice. Without communication, the nursing profession could not exist with its present dimensions [31, 32].

\section{Conclusions}

Pain is a universal experience and an unavoidable part of human being. The role of health professionals, such as nurses, is very important in the treatment of pain. They have to take care of people who are in pain, as well as to respect each individual's personality and beliefs. Moreover, parallel to the medical treatment administered to the patient who is in pain, they try to use the appropriate means to help the patient in 
reducing the anxiety and phobias that increase pain and affect their everyday quality of life. In order to achieve this goal, nurses should be well educated and specialized in pain management. Pain management nurses assess patients to determine the severity and causes of their pain and use adequate techniques to relieve it. Some of these techniques may include biofeedback, acupuncture, massage and therapeutic exercises. It is also important written evidence-based recommendations to be given to nurses and other members of the inter-professional team who are assessing and managing people with pain.

\section{References}

[1] Kostopoulos, K. 2011. "The Psychosomatic Pain." Achaiki Iatriki 30 (2): 163-6.

[2] Armeni, K. 2010. "The History of Analgesia in Greece, from Antiquity to Today." Doctoral thesis, Department of Philosophy, Education and Psychology, University of Ioannina, Greece.

[3] Zois, K., and Patiraki-Kourbani, E. 2008. "The Culture of Health Professionals and Suboptimal Treatment of Pain." Nursing 47: 167-74.

[4] Sarakatsianou, Ch. 2012. "Assessment and Suboptimal Treatment of Postoperative Pain: Myth or Reality?." Vima Asclipiou 11 (2): 162-4.

[5] IASP (International Association for the Study of Pain). 1986. "Subcommittee on Taxonomy." Pain 3: 216-21.

[6] Pefanis, P. 2011. "Texts for Pain and Suffering. Pain as a Biopsychosocial Conceptual Approach.” Synapsis 7 (23) 37-42.

[7] Amprachim, S. E., Lepetsos, P., and Kourkouta, L. 2014. "Causes of Acute Pain Undertreatment." Scientific Chronicles 19 (1): 43-9.

[8] Zuccaro, S. M., Vellucci, R., Sarzi-Puttini, P., Cherubino, P., Labianca, R., and Fornasari, D. 2012. "Barriers to Pain Management: Focus on Opioid Therapy." Clin. Drug Investig. 32: 11-9.

[9] Breivik, H., Borchgrevink, P. C., Allen, S. M., Rosseland, L. A., Romundstad, L., Breivik Hals, E. K., et al. 2008. “Assessment of Pain.” British Journal of Anaesthesia 101 (1): 17-24.

[10] Katsimigas, G., Spiliopoulou, C., Nastou, C., and Gika, M. 2009. "Nursing and Theological Approach of Pain." Vima Asklipiou 8 (2): 159-76.

[11] Kourkouta, L. I. 2010. Diagnostic Nursing Approach. Athens: P.C. Paschalidis.

[12] Tsakopoulos, I. 2001. Physiology of Man-Mechanisms of Body's Functioning. 8th ed. Athens: Pashalidis.
[13] Iatrou, Ch. 2003. "Everything We Have to Know about Nociceptive Pain." Greek Magazine of Perioperative Medicine 1: 48-57.

[14] Goules, D. 2008. "Semantics of Pain. The Pain as an Experience and Behavior." InfoOrthopaedics \& Traumatology 50: 12-5.

[15] Akirou, D. 1994. "Evaluation of the Pain of Patients Suffering from Acute Myocardial Infarction.” Doctoral thesis, Nursing Department, EKPA, Athens, Greece.

[16] Giala, M. 2002. "The Doctor Facing the Incurable Pain and Death. Analgesic Treatment for Sufferers from Unresectable Disease." Presented at Scientific Symposium "Euthanasia”, Papageorgiou Hospital, Thessaloniki, Greece.

[17] Argyra, E., Vadalouka, A., Siafaka, I., and Papadopoulos, G. 2006. Treatment of Acid and Chronic Pain. Ioannina: Efyra.

[18] Yfantopoulos, J., Papagiannopoulou, V., and Papanikolaou, S. 2010. "Determination of the Greek Clinical Practice for the Management of Post-operative Pain." Archives of Hellenic Medicine 27 (4): 650-61.

[19] Runge, M., and Greganti, M. A. 2015. Netter Pathology. 2nd ed. Athens: Broken Hill Publishers Ltd.

[20] Papandreou, Ch., and Damigos, D. 2008. "The Treatment of Chronic Pain in the Elderly." Medical Subject May-June: 36-42.

[21] Namukwaya, E., Leng, M., Downing, J., and Katabira, E. 2011. "Cancer Pain Management in Resource-Limited Settings: A Practice Review." Pain Res. Treat. 2011: 393-404.

[22] Jacobsen, R., Liubarskiene, Z., Moldrup, C., Christrup, L., Sjøgren, P., and Samsanaviciene, J. 2009. "Barriers to Cancer Pain Management: A Review of Empirical Research." Medicina (Kaunas) 45: 427-33.

[23] Patiraki, E. 1995. "Nursing Assessment Sick Cancer Pain." Doctoral thesis, Nursing Department, EKPA, Athens, Greece.

[24] Edmunds, M. 2003. Introduction to Clinical Pharmacology. 1st edn. Athens: Parisianou A. E.

[25] Ferrel, B. A. 2001. "Pain Management in the Elderly." Clin. Geriatr. Med. 17: 417-615.

[26] Momeni, M., Crucitti, M., and De Kock, M. 2006. Patient-Controlled Analgesia in the Management of Postoperative Pain.” Drugs 66: 2321-37.

[27] Aretha, D. 2011. "Optimized Perioperative Analgesia Reduces Chronic Phantom Limb Pain Intensity, Prevalence, and Frequency: A Prospective, Randomized, Clinical Trial." Ph.D. thesis, Department of Medicine, SEYP, University of Patra, Patra, Greece.

[28] Ragia, A. 2002. Basic Nursing-Theoretical and Ethical Principles. 5th ed. Athens: Ragia.

[29] Katz, B., and Helme, R. 2003. "Pain in Old Age." In Brocklehurst's Textbook of Geriatric Medicine and 
Gerontology, edited by Tallis, R. C., and Fillit, H. M. 6th ed. London: Churchill Livingstone, 1391-406.

[30] Fradelos, E., Fradelou, G., and Kasidi, E. 2014. "Pain: Aspects and Treatment in Greek Antiquity.” Journal of Medical Sciences and Public Health 2 (2): 29-36.
[31] Kourkouta, L., and Papathanasiou, I. V. 2014. “Communication in Nursing Practice." Mater. Sociomed. 26 (1): 65-7.

[32] Papadantonaki, A. 2006. "Communication and Nursing." Nursing 45 (3): 297-8. 